\title{
Experimental Tests of Cooling: Expectations and Additional Needs
}

\author{
Michael S. Zisman ${ }^{1,2}$ \\ Lawrence Berkeley National Laboratory \\ One Cyclotron Road, Mail Stop 71R0259, Berkeley, CA 94720, U.S.A. \\ E-mail: mszismanelbl.gov
}

\begin{abstract}
Cooling is a critical aspect for a high-performance Neutrino Factory or a Muon Collider. For this reason, considerable effort is being put toward the experimental verification of this technique. The international Muon Ionization Cooling Experiment, MICE, was approved to operate at Rutherford Appleton Laboratory (RAL) in the UK and beam line commissioning commenced in March, 2008. The MICE collaboration comprises about 130 scientists and engineers from Asia, Europe, and the U.S. In this paper we present the motivation and goals for this experiment and describe its present status. MICE is scheduled for completion in 2011. We will also indicate the prospects for a future 6D muon cooling experiment and discuss its possible time schedule.
\end{abstract}

10th International Workshop on Neutrino Factories, Super beams and Beta beams Valencia, Spain

30 June - 05 July, 2008

\footnotetext{
${ }^{1}$ Speaker

${ }^{2}$ This work was supported by the Director, Office of Science, Office of High Energy Physics, of the U.S. Department of Energy under Contract No. DE-AC02-05CH11231.
} 


\section{Introduction}

Muon beam accelerators have the potential to address the main outstanding acceleratorrelated questions in particle physics. In the neutrino sector, a Neutrino Factory based on a stored muon beam can provide well-characterized beams of high energy neutrinos and anti-neutrinos via the decays

$$
\mu^{+} \rightarrow e^{+} v_{e} \bar{v}_{\mu} \text { and } \mu^{-} \rightarrow e^{-} \bar{v}_{e} v_{\mu} .
$$

The kinematics of these decays is well known and there are no hadronic uncertainties in the spectrum and flux. Decays of $\mu^{+}$give a neutrino beam with $50 \% v_{e}$ and $50 \%$ anti- $v_{\mu}$, and decays of $\mu^{-}$give a neutrino beam with $50 \% v_{\mu}$ and $50 \%$ anti- $v_{e}$. A muon-based Neutrino Factory is the most effective tool to probe the neutrino sector and, hopefully, observe CP violation in leptons. Results from such a facility will test theories of neutrino masses and oscillation parameters, whose predictions are important to both particle physics and cosmology.

At the energy frontier, the fact that the muon is a point particle (as opposed to the composite nature of the proton) means that the full collision energy is available for producing new particles. In addition, the muon, being much heavier than the electron, emits negligible synchrotron radiation. This has two beneficial consequences for a Muon Collider:

- muons can be stored in a circular collider fitting on an existing laboratory site

- beamstrahlung is suppressed, resulting in a narrow energy spread for collisions.

These features make muon beams very attractive to consider for use in particle physics but, as will be discussed below, producing such beams is very challenging.

\section{Why Cool?}

The need for cooling arises from the fact that muons are created as a tertiary beam, and thus initially have a large energy spread and a very large transverse phase space. The beam emittance is simply too large for efficient transport in cost-effective accelerators, so a means to increase the phase-space density is required. Although there are a number of established techniques for cooling a beam, the choice for muon cooling is restricted because of the short muon lifetime ( $2.2 \mu \mathrm{s}$ at rest). Indeed, only ionization cooling is a viable technique.

The large initial phase space has another consequence. It means that solenoidal transport lines, which focus in both planes simultaneously, are preferred. The short lifetime similarly has consequences, in particular putting a substantial premium on rapid beam manipulations and thus on the highest practical RF gradients.

\section{The Muon Ionization Cooling Experiment (MICE)}

A high-performance Neutrino Factory, producing $\sim 10^{21} v_{e}$ aimed at a far detector per $10^{7}$-s year, depends on ionization cooling. ${ }^{1}$ The physics of ionization cooling is straightforward, but

\footnotetext{
${ }^{1}$ A Muon Collider depends even more heavily on ionization cooling, so an experimental test of the technique is motivated by both types of facility.
} 
the implementation is not, and the technique has not yet been experimentally demonstrated. Since a Neutrino Factory is expected to be expensive, it is highly appropriate to test its key principle. This is the motivation for MICE.

The MICE experiment aims to

1. design, engineer, and fabricate a section of cooling channel capable of giving the desired performance for a Neutrino Factory

2. place this apparatus in a muon beam and measure its performance in a variety of operating modes and beam conditions

3. show that our simulation codes agree with experiment.

The last goal validates our design tools, giving confidence that we can ultimately optimize the design of an actual facility. For this to succeed, it is important that both the apparatus and the simulations be as realistic as possible.

\subsection{System Description}

MICE includes one cell of the so-called Feasibility Study 2 [1] cooling channel. As can be seen in Fig. 1, the cooling cell comprises three focus coil (FC) modules with absorbers $\left(\mathrm{LH}_{2}\right.$ or solid) and two RF-coupling coil (RFCC) modules, each with four 201-MHz RF cavities. To complete the experimental apparatus, we have two spectrometer solenoids (one upstream and one downstream of the cooling cell) housing scintillating fiber tracking detectors, along with other detectors (time-of-flight, Cherenkov, and calorimeter) to perform particle identification.

The focus coil module, a responsibility of RAL and University of Oxford, is being designed in industry. It comprises two coils that can be operated with either the same or opposite polarities. A $20-\mathrm{L} \mathrm{LH}_{2}$ absorber (provided by KEK) fits inside each of the three modules. The $\mathrm{LH}_{2}$ handling system (provided by RAL) uses a commercial metal-hydride bed as its storage system. The design has passed two international safety reviews and an R\&D system is presently being built in industry. The absorber makes use of thin $(125 \mu \mathrm{m})$ aluminum windows, $300 \mathrm{~mm}$ in diameter (provided by U.-Mississippi). Sample windows have already been tested to a burst pressure of nearly 10 bar.

The RFCC module, illustrated in Fig. 2, is designed and initial fabrication has begun. The $\mathrm{CC}$ magnets are being fabricated by the Institute of Cryogenics and Superconductivity Technology of the Harbin Institute of Technology in China and the cavities by LBNL.

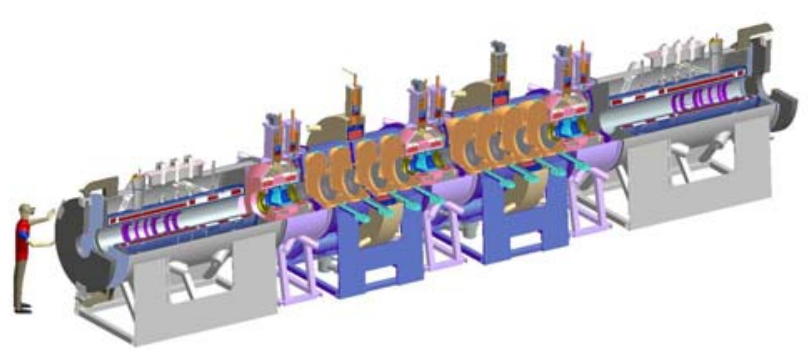

Figure 1: Layout of MICE experiment.

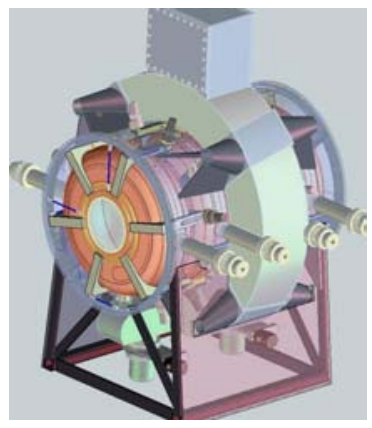

Figure 2: RFCC module comprising four RF cavities and one CC. 
The two spectrometer solenoids (provided by LBNL) are being fabricated in industry, and the first device is nearly complete. The first of two tracker detectors (provided by a number of UK groups led by Imperial College along with groups from Osaka U., IIT, and Fermilab) is completed and under test at RAL; fabrication of the second unit is well along.

The detectors for particle identification come from many groups. The TOF systems are provided by INFN-Milano and U.-Geneva, the Cherenkov detectors by U.-Mississippi, and the calorimeter by INFN-Roma3, U.-Geneva, and INFN-Trieste U.

At a typical muon momentum of $200 \mathrm{MeV} / \mathrm{c}$, we expect about a $10 \%$ emittance reduction, and MICE is designed to measure emittance with a precision of $10^{-3}$. By making a "virtual scan" over the input emittance (from $\sim 1-10 \pi \mathrm{mm}$-rad) and observing the corresponding change in emittance after the cooling cell, the experiment can determine the equilibrium emittance of the channel, expected to be about $2.5 \pi \mathrm{mm}$-rad.

\section{Future Activities}

By the time MICE has successfully concluded, we will have demonstrated an understanding of the 4D (transverse) cooling process and an understanding of the technical challenges that must be met, both in terms of component design and in terms of system operation. This information is expected to be sufficient for developing a Neutrino Factory reference design report (RDR), with the possible exception of the need to demonstrate alternative RF approaches, such as magnetically insulated cavities.

Two tasks are missing from the collider effort. One is an equivalent demonstration of $6 \mathrm{D}$ cooling, and the other is a demonstration of the final transverse cooling. An obvious question with regard to these items is whether they are needed in order to design the facility or only to get it approved for funding. ${ }^{2}$

\subsection{Other 6D Cooling Component Tests}

Before proceeding to a realistic $6 \mathrm{D}$ cooling experiment, substantial component $\mathrm{R} \& \mathrm{D}$ must be carried out. Most critical is development of the means to avoid — or at least mitigate - the observed degradation of RF gradient in a strong magnetic field. Palmer [2] has suggested the idea of using a magnetically insulated open-cell cavity for this purpose, and has proposed the test system illustrated in Fig. 3. It is likely that some variant of this test will be done in the U.S.

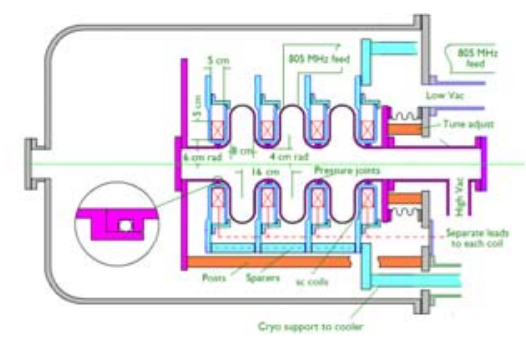

Figure 3: Proposed magnetic insulation test setup for $805-\mathrm{MHz}$ cavities. Such a test, if successful, would be followed by a test of a full-size 201-MHz cavity system.

\footnotetext{
${ }^{2}$ The tacit assumption currently is that these demonstrations can be done after the design.
} 
There are two other items required for a Muon Collider cooling system-wedge-shaped absorbers and high field solenoids $(\sim 50 \mathrm{~T})$ for the final cooling segment. The latter will involve a substantial development program, planning for which is just getting under way.

\subsection{D Cooling at RAL}

The existence of a well-characterized muon beam line at RAL will be valuable for the particle physics community even after MICE is completed. In particular, utilizing this facility for a subsequent $6 \mathrm{D}$ muon cooling experiment is a potentially attractive option. It will be interesting to consider a test of $6 \mathrm{D}$ cooling components, such as a wedge-shaped absorber, with the MICE apparatus. More substantial tests of 6D cooling channels based on a Guggenheim lattice [3], a helical channel [4], or a so-called FOFO snake [5] are also worthy of consideration, once the best arrangement has been determined.

It seems impractical to contemplate launching a full 6D experiment much before 2012, but that time scale should nonetheless permit completion of the experiment in time for preparation of a Conceptual Design Report for a Muon Collider starting in the middle of that decade.

\section{Summary}

The MICE experiment is making good progress and should be able to answer most questions about transverse (4D) cooling in the next few years. Demonstration of 6D cooling is expected to require a significant component-development effort, followed by a substantial international experiment. Adopting the Guggenheim or FOFO-snake scheme would likely reduce the cost of the experiment, as the components are similar to those already in use for MICE. The strong R\&D program to develop and test key cooling channel components must continue, and even expand, if we are to complete the work in a timely way.

\section{References}

[1] S. Ozaki, R. Palmer, M. Zisman, and J. Gallardo (eds.), Feasibility Study-II of a Muon-based Neutrino Source, BNL-52623, June, 2001, see: http:/www.cap.bnl.gov/mumu/studyii/final_ draft/The-Report.pdf.

[2] R. Palmer, R. Fernow, and J. Gallardo, Magnetic Insulation of RF, see http://www.fnal.gov/projects/muon_collider/FridayMeetings/23-MAY-2008/Palmer.pdf.

[3] A. Klier, How to Guggenheim the RFOFO Ring, in Proc. Low Emittance Collider Workshop, 2006, http:/www.muonsinc.com/mcwfeb06/presentations/AKlier_02072006_Guggenheim.pdf.

[4] Y. Derbenev and R. P. Johnson, Six-dimensional Muon Beam Cooling Using a Homogeneous Absorber, Phys. Rev. ST-AB 8, 041002 (2005).

[5] Y. Alexahin, K. Yonehara, and R. Palmer, 6D Ionization Cooling Channel with Resonant Dispersion Generation, http://accelconf.web.cern.ch/AccelConf/p07/PAPERS/THPAN106.PDF. 